\title{
Le retard de maturation visuelle; un cas clinique
}

\author{
PAR MARIE-EVE CORBEIL, OD, MSC
}

\author{
RÉSUMÉ \\ Lors de son premier examen visuel \\ complet, le patient de deux mois et demi \\ ne fixe pas et ne suit pas la cible ou la \\ lumière présentée et ce, malgré un \\ examen visuel normal. Cette condition \\ temporaire est appelée retard de \\ maturation visuelle. \\ CONCLUSION : À cinq mois et demi, le \\ comportement visuel de l'enfant s'est nor- \\ malisé. Le retard de maturation visuelle \\ est un diagnostic d'exclusion qui nécessite \\ un examen oculo-visuel complet, des \\ tests électrodiagnostiques et si requis, \\ d'imagerie par résonance magnétique.
}

\section{Introduction}

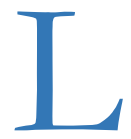

a suspicion d'une vision anormale chez un nouveau-né doit être prise très au sérieux. En effet, dans $80 \%$ des cas, le professionnel découvre à l'examen une cause évidente de déficit visuel comme une cataracte, du glaucome ou de l'albinisme. ${ }^{1}$ Parfois, comme dans l'amaurose congénitale de Leber, il s'agit plutôt de signes sur la rétine, d'anomalies pupillaires ou de nystagmus. D'autres anomalies, comme la cécité corticale, sont accompagnées de troubles neurologiques importants. Par contre s'il s'agit d'un simple retard dans la maturation visuelle, tous les tests objectifs peuvent être normaux, ce qui complique le diagnostic. Les tests électrodiagnostics sont alors très utiles pour faire le diagnostic différentiel entre les différentes conditions et orienter les parents sur l'aspect permanent ou temporaire de la basse vision.

\section{Exposé de cas}

A.J., un garçon de deux mois et demi, est amené par sa mère pour un examen visuel complet. Cette dernière a remarqué qu'A.J. semble avoir un problème de vision puisqu'il ne réagit pas aux stimuli visuels. Il répond par un sourire aux voix humaines, mais ne réagit pas aux visages. L'histoire d'A.J. est sans autre particularité : grossesse à terme sans complication, bonne santé, pas d'antécédent familial de problème systémique ou oculaire. À l'examen, l'acuité visuelle par regard préférentiel, l'habileté à regarder et à suivre une lumière ou un gros objet, la présence ou l'absence de nystagmus, les réflexes pupillaires, l'examen externe, le fond d'œil à l'ophtalmoscopie directe et indirecte et la réfraction sous cycloplégie sont évalués (tableau 1). Aucune anomalie particulière n'a été détectée.

Compte tenu du jeune âge du patient et de l'absence de pathologie oculaire visible, un suivi dans un mois et demi est alors recommandé. Lors de ce suivi, les mêmes résultats sont trouvés à l'examen.

Quel est le diagnostic le plus probable?

TABLEAU 1 : Tests et résultats de l'examen visuel d'A.J.

\begin{tabular}{|l|l|}
\hline Tests & Résultats \\
\hline Acuité visuelle (Carte de Teller) & Nil \\
\hline Fixation & Absente \\
\hline Motilités & Nil \\
\hline Nystagmus & Absent \\
\hline Réflexes pupillaires & Normaux \\
\hline Examen externe & Normal \\
\hline Fond d'œil & NO, rétine et macula normaux \\
\hline Réfraction (rétinoscopie sous cycloplégie) & OD $+1,25 /-0,50 \times 180$ \\
& OS $+1,50$ \\
\hline
\end{tabular}




\section{Discussion}

Un enfant présentant un retard de maturation visuelle (RMV) ne fixe pas ou ne suit pas une cible ou une lumière et ce, malgré un examen visuel qui peut être entièrement normal. Dans la plupart des cas, il ne clignera pas à l'approche d'une lumière forte ou d'un objet. Normalement, le réflexe de fixation peut être observé chez l'enfant dès 32 semaines de gestation. ${ }^{2,3}$ À cet âge, le regard préférentiel est aussi présent. ${ }^{4}$ En effet, les nouveau-nés vont préférer regarder un patron ou une cible en mouvement plutôt qu'une plage uniforme. Les poursuites horizontales sont présentes dès la naissance, mais les poursuites verticales se développent entre quatre à six semaines d'âge. Typiquement, les patients atteints de RMV ont entre deux et quatre mois et sont en bonne santé. Ils vont généralement commencer à fixer et à suivre les objets vers l'âge de six mois. Le RMV n'est pas une condition nouvelle. Déjà, en 1926, Beauvieux décrivait des enfants réagissant peu ou pas aux stimuli visuels à la naissance. ${ }^{5}$ Depuis, plusieurs auteurs ont décrit des cas similaires en utilisant différents termes : myelogenosis retardata, développement visuel dissocié ou délai de développement visuel ${ }^{6,7}$ Illingworth (1961) serait le premier à avoir introduit le terme retard de maturation visuelle. Il a décrit la condition de deux bébés, sans aucun retard développemental, dont la réaction normale aux stimuli visuels ne s'est pas manifestée avant l'âge de six mois. ${ }^{8}$ En 1981, Uemera et al. ont présenté une classification des RMV comprenant trois

TABLEAU 2 : Types de RMV ${ }^{9}$

\begin{tabular}{|l|l|}
\hline Types & Caractéristiques \\
\hline 1 & RMV isolé \\
\hline 2 & RMV et retard mental ou épilepsie \\
\hline 3 & RMV et anomalies visuelles primaires \\
\hline
\end{tabular}

\section{TABLEAU 3 : Types de RMV ${ }^{10}$}

\begin{tabular}{|l|l|l|l|}
\hline Types & Caractéristiques & $1 \mathrm{a}$ & Période périnatale normale \\
\hline 1 & RMV est l'anomalie principale & $1 \mathrm{~b}$ & Période périnatale anormale \\
\hline 2 & RMV et trouble de développement neurologique durable \\
\hline 3 & RMV, nystagmus et albinisme \\
\hline 4 & RMV et troubles oculaires congénitaux bilatéraux \\
\hline
\end{tabular}

catégories (tableau 2). ${ }^{9}$ Fielder et al. ont modifié cette classification en proposant plutôt quatre types avec sous catégories (tableau 3). ${ }^{10}$

Le RMV est un diagnostic d'exclusion qui nécessite un examen oculo-visuel complet, des tests électrodiagnostiques et éventuellement d'imagerie par résonance magnétique (IRM). Le recours aux potentiels évoqués visuels (PEV) est fréquent. Les résultats sont toutefois très variables d'une étude à l'autre. Certaines études ont rapporté que les enfants avec RMV montraient aussi une atteinte initiale des PEV. Les PEV flash peuvent être absents ${ }^{7}$, montrer une latence $^{11}$ ou une forme anormale ${ }^{10}$ dans les premiers mois de vie avec une amélioration progressive par la suite. Les PEV pattern montrent des similitudes, mais varient beaucoup en fonction du stimulus utilisé. L'électrorétinogramme (ERG) est anormal seulement si le RMV est associé à des anomalies rétiniennes. ${ }^{12}$ Les principaux diagnostics différentiels sont présentés au tableau 4. Toutes ces conditions présentent un tableau clinique initial comparable. Les deux conditions les plus difficiles à éliminer sont l'hypoplasie papillaire bilatérale et la cécité corticale. Dans le premier cas, l'aspect symétrique des nerfs optiques peut faire passer l'atteinte inaperçue; le diagnostic de la seconde condition qui n'est pas toujours facile à éliminer en jeune âge nécessite souvent une IRM.

Le pronostic des enfants ayant un retard de la maturation visuelle est très variable d'une étude à l'autre et dépend beaucoup du type d'atteinte. Plusieurs auteurs rapportent des cas de RMV isolés ${ }^{7}$ 11, 13 tandis que d'autres les retrouvent associés avec d'autres troubles neurologiques. ${ }^{1}$ Les cas isolés vont se normaliser en moyenne vers la $14^{\mathrm{e}}$ semaine selon Fielder $^{10}$ et entre trois et huit mois selon la majorité des auteurs. ${ }^{12,14,15}$ Cette 
TABLEAU 4 : Principaux diagnostics différentiels

\begin{tabular}{|c|c|}
\hline Conditions & Caractéristiques \\
\hline Amaurose congénitale de Leber & $\begin{array}{l}\text { Nystagmus fréquent } \\
\text { Anomalie papillaire } \\
\text { Variation rétinienne } \\
\text { Altération majeure de l'ERG } \\
\text { Déficit total ou important des PEV }\end{array}$ \\
\hline Albinisme & $\begin{array}{l}\text { Nystagmus possible } \\
\text { Fond d'œil peu pigmenté } \\
\text { ERG et PEV normaux } \\
\text { Transillumination irienne permet le } \\
\text { diagnostic }\end{array}$ \\
\hline Dystrophie des cônes & $\begin{array}{l}\text { Altération majeure de l'ERG } \\
\text { Atteinte des PEV } \\
\text { Nystagmus et photophobie } \\
\text { précoces }\end{array}$ \\
\hline Hypoplasie papillaire bilatérale & $\begin{array}{l}\text { ERG normal } \\
\text { PEV +/- atteints } \\
\text { Tomodensitométrie } \\
\text { permet le diagnostic }\end{array}$ \\
\hline Cécité corticale & $\begin{array}{l}\text { ERG normal } \\
\text { Déficit important des PEV } \\
\text { IRM permet le diagnostic }\end{array}$ \\
\hline
\end{tabular}

guérison est rapide. En quelques jours l'enfant s'éveille, s'intéresse et sourit à son entourage. L'examen visuel complet devient alors comparable à celui des autres enfants normaux du même âge. Lorsque le RMV est associé à un retard mental (type 2 selon Fielder), le pronostic est moins bon à cause des anomalies neurologiques associées. Lorsque le RMV est associé à une autre anomalie oculaire comme l'albinisme, l'aniridie ou autre (type 3 et 4 selon Fielder), la vision initiale paraît totalement absente ou plus basse que ce que l'anomalie oculaire trouvée laisse supposer. Cependant, en quelques mois, en moyenne cinq mois, une certaine vision apparaît, correspondant à celle que l'on était en droit d'espérer avec l'anomalie associée. ${ }^{10}$

Plusieurs hypothèses ont été émises dans le passé pour déterminer la cause du RMV. Selon Beauvieux, il était causé par un retard de myélinisation. ${ }^{5}$ Pendant longtemps, les voies visuelles antérieures étaient considérées comme complètement myélinisées dans les premiers mois de vie. ${ }^{16}$ Des études plus récentes montrent que les fibres du nerf otique commencent leur myélinisation à la naissance, mais que le processus peut se poursuivre jusqu'à l'âge de deux ans. ${ }^{17}$

Selon Hoyt, le RMV était plutôt causé par un retard de maturation de certaines fonctions corticales ${ }^{18}$, tandis que Tresidder croit à une anomalie du système extra géniculé prédominant dans les deux premiers mois de la vie tandis que les fonctions purement corticales émergent normalement après ce délai. ${ }^{19}$

\section{Conclusion}

À l'âge de cinq mois et demi, A.J. est examiné de nouveau. Il présente un comportement visuel à l'intérieur des limites de la normale pour un enfant de son âge. La mère a remarqué qu'il réagit normalement aux stimuli visuels.

Le RMV est une condition visuelle relativement rare dans laquelle l'enfant ne réagit pas à des stimuli visuels, mais ne présente aucune anomalie visible. Le RMV peut se retrouver de façon isolée ou accompagner d'autres anomalies. Dans la majorité des cas, le comportement visuel redevient normal vers l'âge de six mois. Après cet âge, ou si les parents sont inquiets, il est recommandé de faire passer des tests électrodiagnostics (PEV et ERG) pour éliminer toute autre cause de déficit visuel. 


\section{Références}

1. Cole GF, Hungerford J, Jones RB. Delayed visual maturation. Archives of Disease in Childhood 1984;59(2):107-110.

2. Vehrs S, Baum D. A test of visual responses in the newborn. Dev Med Child Neurol 1970;12(6):772-774.

3. Wolff PH, Ferber R. The development of behavior in human infants, premature and newborn. Annu Rev neurosci 1979;2:291-307.

4. Dubowitz L. A study of visual function in the premature baby. Child Care Health Dev 1979;5(6):399-404.

5. Beauvieux J. La pseudo-atrophie optique des nouveaux-nés (disgénésie myélinique des voies optiques). Ann d'oculiste 1926;163:876-921.

6. Keiner GBJ. New viewpoints on the origin of squint. Br J Ophthalmol 1952;36(6)334.

7. Mellor DH, Fielder AR. Dissociated visual developmet, electrodiagnostic tests in infants who are "slow to see". Dev. Med child Neurol 1980;22(3):327-335.
8. Illingworth RS. Delayed visual maturation. Arch Dis Child 1961;36:407-409.

9. Umera Y, Agucci Y, Katsumi, O. Visual development delayed. Ophthalmic Paediatrics and Genetics 1981;1:4-11.

10. Fielder AR et al. Delayed visual maturation. Trans Ophthalmol Soc UK 1985;104(6):653-661.

11. Harel S, Holtzman M, Feinsod M. Delayed visual maturation. Arch Dis Child 1983;58(4):298-299.

12. Mercuri E, et al. The aetiology of delayed visual maturation: short review and personal findings in relation to magnetic resonnance imaging. Euro Paediatric Neurol 1997;1(1):31-34.

13. Dudgeon J. Electrodiagnostic tests in visual assessment in children. Dispensing Optician 1977;29:222-229.

14. Lambert SR, Kriss A, Taylor D. Delayed visual maturation. A longitudinal clinical and electrophysiological assessment. Ophthalmology. 1989;96(4):524-529.
15. Harris $\mathrm{CM}$ et al. Delayed visual maturation in infants: A disorder of figure-ground separation? Brain Reserch Bulletin 1996;40(56):365-369.

16. Carvioto H. Electron microscopic studies of the developing human nervous system. II. The Optics nerve. J Neuropathol Exp Neurol 1965;24:166-167.

17. Maggon EH, Robb RN. Development of myelin ine the human optic nerve and track. Arch Ophthalmol 1981;99:665-669.

18. Hoyt CS. Constenbader lecture. Delayed visual maturation: the apparently blind infant. Journal of American Association for pediatric Ophthalmology and Strabismus 2004;8 $\operatorname{Vol}(3): 215-219$.

19. Tresidder J, Fielder AR, Nicholson J. Delayed visual maturation: ophthalmic and neurodevelopmental aspects. Developmental medicine and child neurology. 1990;32(10): 372-381.
Are you doing all you can to maximize the revenue potential of your practice?

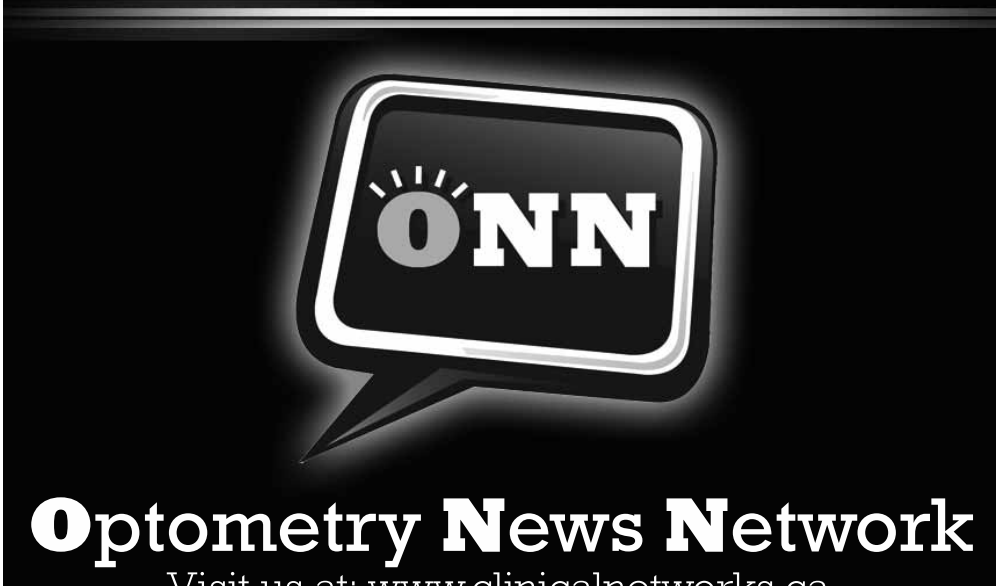

Visit us at: www.clinicalnetworks.ca
National Glaucoma Society

National Cornea and Anterior Segment Society Continuing Education Programs

Manchester, NH November 8, 2009

Marlborough, MA December 6, 2009

Orlando, FL

Falls Church, VA

January 17,2010

Cape Cod, MA

March 14, 2010

July 24-26, 2010

Live Webinars available from all conferences

Conference Registration: www.neconferences.com

Toll-free: 877-825-2020 